TECHNICAL TRANSACTIONS 7/2018

MATHEMATICS

DOI: $10.4467 / 2353737$ XCT.18.106.8801 SUBMISSION OF THE FINAL VERSION: 24/04/2018

Andrzej Tomski (andrzej.tomski@us.edu.pl)

Institute of Mathematics, Faculty of Mathematics, Physics and Chemistry, University of Silesia

Maciej Zakarczemny (mzakarczemny@pk.edu.pl)

Institute of Mathematics, Faculty of Physics, Mathematics and Computer Science,

Cracow University of Technology

\title{
A NOTE ON BROWKIN'S AND CAO’S CANCELLATION ALGORITHM
}

UWAGI O ALGORYTMIE SITOWYM BROWKINA I CAO

\begin{abstract}
In this paper, we follow our generalisation of the cancellation algorithm described in our previous paper [A. Tomski, M. Zakarczemny, On some cancellation algorithms, NNTDM. 23, 2017, p. 101-114]. For $f$ being a natural-valued function defined on $\mathbb{N}^{s}, s \geq 1$ we remove the divisors of all possible values of $f$ in the points in which the sum of coordinates is less than or equal to $n$. The least non-cancelled number is called the discriminator $D_{f}(n)$. We find formulas, or at least an estimation for this discriminator, in the case of a broad class of sequences.
\end{abstract}

Keywords: discriminator, sequence, congruence, odious numbers, Thue-Morse sequence

\section{Streszczenie}

Kontynuujemy badania nad generalizacją algorytmu sitowego Browkina i Cao, [A. Tomski, M.Zakarczemny, On some cancellation algorithms, NNTDM. 23, 2017, p. 101-114]. Niech $f$ będzie funkcją o wartościach w zbiorze liczb naturalnych, określoną na $\mathbb{N}^{s}, s \geq 1$. Usuwamy dzielniki wszystkich możliwych wartości funkcji $f$, w punktach, w których suma współrzędnych nie przekracza $n$. Najmniejszą niewykreśloną liczbę naturalną nazywamy dyskryminatorem $D_{f}(n)$. W artykule uogólniamy pojęcie dyskryminatora. Znajdujemy jawne wzory lub oszacowania na dyskryminator dla szerokiej klasy ciągów.

Słowa kluczowe: dyskryminator, ciąg, kongruencja, liczby wstrętne, ciąg Thue-Morse’a 


\section{Introduction}

Let

$$
D_{g}(n):=\min \{m \in \mathbb{N}: g(1), g(2), \ldots, g(n) \text { are different modulo } m\}
$$

for some special injective function $g: \mathbb{N} \rightarrow \mathbb{N}$.

The problem is to find $D_{g}(n)$ for an at least sufficiently large $n \in \mathbb{N}$.

We call $D_{g}(n)$ the discriminator of the function $g$ as it gives the least modulus which discriminates the exact value of $g$, i.e. the $n$ numbers $g(1), g(2), \ldots, g(n)$ are pairwise incongruent modulo $D_{g}(n)$.

This idea was first introduced in [1] in the case of $g(n)=n^{2}$ and through the years has remained an object of interest for many number theorists. The authors of [2] solved the problem for $g(n)=n^{j}$ where $j \in \mathbb{N}$ and $n$ is sufficiently large. In [5], the Dickson discriminator problem was considered - this is to find $D_{g}(n)$ for a Dickson polynomial of degree $j \geq 1$, which is defined by the following formula:

$$
g(x)=\sum_{i=0}^{[j / 2]} \frac{j}{j-i}\left(\begin{array}{c}
j-i \\
i
\end{array}\right)(-a)^{i} x^{j-2 i},
$$

for some integer number $a$. Afterwards, [6] and [13] provided an asymptotic characterisation of $D_{g}(n)$ for some special $g \in \mathbb{Z}[x]$ including cyclic polynomials. In turn, Zhi-Wei Sun found such examples of $g$ that for any $n$, the value of $D_{g}(n)$ is a prime.

Example 1.1. Let $g(n)=n-(-1)^{n}$ where $g: \mathbb{N} \rightarrow \mathbb{N}$. Then $D_{g}(n)=2\left[\frac{n+1}{2}\right], n \geq 2$.

Proof. By straightforward verification, we get $D_{g}(2)=2$ and $D_{g}(3)=4$.

Let us assume that $n \geq 3$.

If $2 m<n$, then $g(2 m+1) \equiv g(1)(\bmod 2 m)$ and both $1,2 m+1 \in\{1,2, \ldots, n\}$, so $2 m \neq D_{g}(n)$. If $2 m+1 \leq n$, then $g(2 m+1) \equiv g(2)(\bmod 2 m+1)$ and both $2,2 m+1 \in\{1,2, \ldots, n\}$, so $2 m+1 \neq D_{g}(n)$. Hence,

$$
D_{g}(n) \geq\left\{\begin{array}{c}
n, \text { if } n \text { is even, } \\
n+1, \text { if } n \text { is odd }
\end{array}\right.
$$

We have two cases.

I. $n$ is odd. If there exist $n_{1}, n_{2} \in\{1,2, \ldots, n\}, n_{1}<n_{2}$ such that

$$
g\left(n_{2}\right) \equiv g\left(n_{1}\right)(\bmod n+1), \text { then } g\left(n_{2}\right) \equiv g\left(n_{1}\right)(\bmod 2), n_{2} \equiv n_{1}(\bmod 2) \text {, }
$$

thus, $g\left(n_{2}\right)-g\left(n_{1}\right)=n_{2}-n_{1}$. Therefore $n+1 \mid n_{2}-n_{1}$, which is not possible.

We have shown that $g(1), g(2), \ldots, g(n)$ are different modulo $n+1$.

Thus, $D_{g}(n) \leq n+1$ and in this case, we have $D_{g}(n)=n+1$. 
II. $n$ is even. Analogically, if there exist $n_{1}, n_{2} \in\{1,2, \ldots, n\}, n_{1}<n_{2}$ such that $g\left(n_{2}\right) \equiv g\left(n_{1}\right)(\bmod n)$, then $n \mid n_{2}-n_{1}$ and we obtain contradiction again.

We have shown that $g(1), g(2), \ldots, g(n)$ are different modulo $n$.

Thus, $D_{g}(n) \leq n$, so in this case, we have $D_{g}(n)=n$.

To summarise, we obtain: $D_{g}(n)=2\left[\frac{n+1}{2}\right]$.

Browkin and Cao [3] reformulated the problem (1) in terms of the following cancellation algorithm. For $n \geq 2$ define the set:

$$
A_{g}(n):=\{g(s)-g(r): 1 \leq r<s \leq n\}=\{g(k+l)-g(l): k+l \leq n ; k, l \in \mathbb{N}\} .
$$

Cancel in $\mathbb{N}$ all numbers from the set:

$$
\left\{d \in \mathbb{N}: d \mid a \text { for some } a \in A_{g}(n)\right\},
$$

we are then interested in finding the least non-cancelled number.

To generalize, let $f: \mathbb{N}^{m} \rightarrow \mathbb{N}, m \geq 1$ be an arbitrary function and define the sets:

$$
\begin{gathered}
V_{f}(n)=\left\{f\left(n_{1}, n_{2}, \ldots, n_{m}\right): n_{1}+n_{2} \cdots+n_{m} \leq n\right\}, \\
\Delta_{f}(n)=\left\{d \in \mathbb{N}: d \mid a \text { for some } a \in V_{f}(n)\right\} .
\end{gathered}
$$

Definition 1.1. We define $b_{f}(n)$ as the least number in the set $\mathbb{N} \backslash \Delta_{f}(n)$ being called the set of all non-cancelled numbers.

Remark. We would like to stress that for any $n \in \mathbb{N}$, the definitions of $D_{g}(n)$ and $b_{f}(n)$ are not equivalent. To be precise, if $g: \mathbb{N} \rightarrow \mathbb{N}$ is an injective function, to find $D_{g}(n)$ is the same task as to find $b_{f}(n)$ with $f\left(n_{1}, n_{2}\right)=g\left(n_{1}+n_{2}\right)-g\left(n_{1}\right)$, see [3].

However, the question is whether, for some given function $f$ having fixed number of variables, there is any injective function $g$, such that $f\left(n_{1}, n_{2}\right)=g\left(n_{1}+n_{2}\right)-g\left(n_{1}\right)$.

In this case, we have $b_{f}(n)=D_{g}(n)$.

Note that the sets of the divisors for both of the values $g\left(n_{1}+n_{2}\right)-g\left(n_{1}\right)$ and $\left|g\left(n_{1}+n_{2}\right)-g\left(n_{1}\right)\right|$ are the same.

Therefore, if for some $n_{1}, n_{2} \in \mathbb{N}, g\left(n_{1}+n_{2}\right)-g\left(n_{1}\right)<0$, we can then take $f\left(n_{1}, n_{2}\right)=\left|g\left(n_{1}+n_{2}\right)-g\left(n_{1}\right)\right|$. For example, in the case $\mathrm{g}(n)=n-(-1)^{n}$, we take $f\left(n_{1}, n_{2}\right)=\left|n_{1}-n_{2}+(-1)^{n_{2}}-(-1)^{n_{1}}\right|$ instead of $n_{1}-n_{2}+(-1)^{n_{2}}-(-1)^{n_{1}}$. The following table shows the connections between these two concepts. 
Table 1. Examples showing that $b_{f}(n)$ generalises $D_{g}(n)$

\begin{tabular}{|c|c|}
\hline $\boldsymbol{b}_{f}(\boldsymbol{n})$ with function $\boldsymbol{f}$ & $\boldsymbol{D}_{\boldsymbol{g}}(\boldsymbol{n})$ with function $\boldsymbol{g}$ \\
\hline$f\left(n_{1}, n_{2}\right)=\left(n_{1}+n_{2}\right)^{2}-n_{1}^{2}$ & $g(r)=r^{2}$ \\
\hline$f\left(n_{1}, n_{2}\right)=\left(n_{1}+n_{2}\right)^{k}-n_{1}^{k}$ & $g(r)=r^{k}, k \in N$ \\
\hline$f\left(n_{1}, n_{2}\right)=k^{n_{1}+n_{2}}-k^{n_{1}}$ & $g(r)=k^{r}, k \in N$ \\
\hline$f\left(n_{1}, n_{2}\right)=\left|n_{1}-n_{2}+(-1)^{n_{2}}-(-1)^{n_{1}}\right|$ & $g(r)=r-(-1)^{r}$ \\
\hline$f(n)=n^{k}$ & - \\
\hline$f\left(n_{1}, n_{2}\right)=n_{1}^{2}+n_{2}^{2}$ & - \\
\hline$f\left(n_{1}, n_{2}, n_{3}\right)=n_{1}^{2}+n_{2}^{2}+n_{3}^{2}$ & - \\
\hline$f\left(n_{1}, n_{2}, \ldots, n_{s}\right)=n_{1} \ldots n_{s} s \in N$ & $g(r)=o d(r)$ \\
\hline$f\left(n_{1}, n_{2}\right)=o d\left(n_{1}+n_{2}\right)-o d\left(n_{1}\right)$ & \\
\hline
\end{tabular}

Browkin and Cao considered standard linear and quadratic functions.

Tomski and Zakarczemny [11] and Zakarczemny [12] investigated the problem mainly in the case of various quadratic and cubic polynomials and also in the case of the products of some linear functions. Now, we provide the formula for $b_{f}(n)$ for new classes of functions.

Haque and Shallit found $D_{g}(n)$ for so-called 'evil' or 'odious' numbers (see [14]); additionally, they counted the number offinite $n$-sequences which are their own discriminators.

2. $f(a)=a$ !

Theorem. Let $f: \mathbb{N} \rightarrow \mathbb{N}, f(a)=a !$. , We have $b_{f}(3)=4$ and:

$$
b_{f}(n)=\min \{p: p>n, p \text { is a prime number }\} \text { if } n \neq 3 .
$$

Proof. By straightforward verification, we may assume that $n>9$. Let $q, p$ be consecutive prime numbers such that $q \leq n<p$. By Bertrand's postulate, we have $2 q>p$.

We note that $p \nmid n !$, hence $p$ is not cancelled.

It is sufficient to show that any natural $m<p$ is cancelled. All the numbers $1,2, \ldots, n$ are cancelled. If we take any $n<m<p$, then $m$ is a composite number.

Let $m=k l$, where $k, l>1$. We investigate two special cases:

I. Let $k=l$ and $k$ be a prime number, so $p>k^{2}>n>9$, so $k \geq 5$. We have:

$$
2 k<\frac{1}{2} k^{2}<\frac{1}{2} p<q \leq n .
$$

As $k<2 k<n$, we get that $2 k^{2} \mid n$ !, so $m \mid n$ ! and $m$ is cancelled. 
II. In any other case, we may assume that $m=k l, k>l>1$. We have:

$$
n>q>\frac{1}{2} p>\frac{1}{2} k l \geq k .
$$

As $l<k<n$ we obtain $k l \mid n !$, so $m \mid n$ ! and $m$ is cancelled.

3. $f(a)=a ! !$

Theorem. Let $f: \mathbb{N} \rightarrow \mathbb{N}, f(a)=a ! !$. We have $b_{f}(7)=b_{f}(8)=9$ and:

if $n \neq 7,8$.

$$
b_{f}(n)=\min \{m: m>n, m=p \text { or } m=2 p, p \text { is } a \text { prime number }\}
$$

Proof. By straightforward verification, we may assume that $n \geq 25$. Let:

$$
T=\{m: m=p \text { or } m=2 p, p \text { is } a \text { prime number }\}
$$

Let $\left(t_{n}\right)_{n=1}^{\infty}$ be an increasing sequence of elements of $T$. There exists $i \in \mathbb{N}$ such that:

$$
t_{i-1} \leq n<t_{i} .
$$

For $i>1$, the following inequality holds:

$$
t_{i}<2 t_{i-1} \text {. }
$$

Equation (11) follows from Bertrand's postulate, which states that for every $x>1$ every interval $(x, 2 x)$ contains at least one prime number. We may assume that $t_{i} \geq 26$.

It is sufficient to show that any natural number $m<t_{i}$ is cancelled.

Let us observe that $m \mid m$ !!, so all the numbers $m \leq n$ are cancelled.

If we take any $m$ such that $n<m<t_{i}$, then $m \in\left(t_{i-1}, t_{i}\right)$, so $m \notin T$ and $m$ is a composite number. Let $m=k l$, where $k, l>1$. We investigate two special cases:

I. Let $k=l$ and $k$ be a prime number, so $t_{i}>k^{2}>n \geq 25$, so $k \geq 7$. We have:

$$
3 k<\frac{1}{2} k^{2}<\frac{1}{2} t_{i}<t_{i-1} \leq n
$$

As $k<3 k<n$, we obtain $3 k^{2} \mid n !$ ! if $n$ is odd or $3 k^{2} \mid(n-1)$ !! if $n$ is even.

Therefore, $m \mid n !$ ! or $m \mid(n-1) ! !$ and $m$ is cancelled.

II. In any other case, we may assume that $m=k l, k>l>1$.

We have the following cases:

a) If $m$ is odd or $4 \mid m$, then we can assume that $k \equiv l(\bmod 2)$. Thus, we have:

$$
n \geq t_{i-1}>\frac{1}{2} t_{i}>\frac{1}{2} k l \geq k .
$$

As $l<k<n$, we obtain $k l \mid n !$ ! if $k \equiv l \equiv n(\bmod 2)$ or $k l \mid(n-1) ! !$ if $k \equiv l \equiv n-1(\bmod 2)$. 
Therefore $m \mid n ! !$ or $m \mid(n-1) ! !$ and $m$ is cancelled.

(b) If $m=2 h$ and $h$ is odd, then $h$ is not a prime since $m \notin T$. Thus, $h=a b, a \geq b \geq 3$.

We can take $k=2 a$ and $l=b$. For $l \geq 5$, we have:

$$
n \geq t_{i-1}>\frac{1}{2} t_{i}>\frac{1}{2} k l \geq k
$$

As $2 l<2 k<n$, we obtain $4 k l \mid n !$ ! if $n$ is even or $4 k l \mid(n-1)$ !! if $n$ is odd.

For $l=3$, we have:

$$
n \geq t_{i-1}>\frac{1}{2} t_{i}>\frac{1}{2} k l \geq k
$$

Since $3 k=k l>n \geq 25$, we may assume that $k>6$. As $6<k<n$, we get that $6 k \mid n$ !! if $n$ is even or $6 k \mid(n-1) ! !$ if $n$ is odd. Therefore, in both cases $m \mid n ! !$ or $m \mid(n-1) ! !$ and $m$ is cancelled. To sum up, any $n<m<t_{i}$ is cancelled, so $b_{f}(n)=t_{i}$ for $n \geq 25$.

4. $f(a)=a 2^{a}$

Theorem 4.1. Let $f: \mathbb{N} \rightarrow \mathbb{N}$, we take the function $f(a)=a 2^{a}$.

Then

$$
b_{f}(n)=2\left[\frac{n+1}{2}\right]+1=\min \{m \in \mathbb{N}: m>n,(m, 2)=1\} .
$$

Proof. Let $k \in \mathbb{N}$ and $n \in\{2 k-1,2 k\}$. If $(2 k+1) \mid f(a)$, then $(2 k+1) \mid a$, so $n+1 \leq 2 k+1 \leq a$, thus $a>n$ and $2 k+1$ is not cancelled. Therefore,

$$
b_{f}(n) \leq 2 k+1
$$

If $1 \leq h \leq n$, then $h \mid f(h), h \leq n$, so $h$ is cancelled and $b_{f}(n) \geq n+1$.

We consider two cases:

1) If $n=2 k$, then $b_{f}(n)=n+1=2\left[\frac{n+1}{2}\right]+1$.

2) If $n=2 k-1$, then $b_{f}(n) \in\{n+1, n+2\}$. If $h=n+1$ then

$h=2 k, h \mid f(k), k=\frac{1}{2} h=\frac{1}{2}(n+1) \leq n$, so $h$ is cancelled and $b_{f}(n)=n+2=2\left[\frac{n+1}{2}\right]+1$.

We generalise this theorem to the following form. 
Theorem 4.2. Let $f: \mathbb{N} \rightarrow \mathbb{N}$ and we fix $b \in \mathbb{N}$. We take the function $f(a)=a b^{a}$.

Then:

if $n>b$.

$$
b_{f}(n)=\min \{m \in \mathbb{N}: m>n,(m, b)=1\},
$$

Proof. Let $m_{0}=\min \{m \in \mathbb{N}: m>n,(m, b)=1\}$.

If $m_{0} \mid f(a)$, then $m_{0} \mid a$, so $n+1 \leq m_{0} \leq a$, thus $a>n$ and $m_{0}$ is not cancelled.

Therefore, $b_{f}(n) \leq m_{0}$.

Let assume that $n>b$. From Bertrand's postulate, it follows that we can find a prime number $p$ such that $n<p<2 n$. Because $p>b$ and $p$ is a prime number, we obtain $(p, b)=1$, so $m_{0} \leq p<2 n$. Thus, $m_{0} \leq 2 n-1$.

If $1 \leq h \leq n$, then $h \mid f(h), h \leq n$, so $h$ is cancelled.

If $n+1 \leq h<m_{0}$, then $(h, b)=d>1$.

Let $h=d l$, where $l \geq 1$, then $h \mid f(l), l=\frac{1}{d} d l \leq \frac{1}{2} d l=\frac{1}{2} h \leq \frac{1}{2}\left(m_{0}+1\right) \leq n$, so $h$ is cancelled and $b_{f}(n) \geq m_{0}$. Therefore, $b_{f}(n)=m_{0}$.

\section{Remark 4.3.}

Let $f: \mathbb{N} \rightarrow \mathbb{N}$ and for all natural $n$ we have $\frac{f(n)}{n} \in \mathbb{N}$, then $b_{f}(n)>n$.

Indeed, if $1 \leq k \leq n$, then $h \mid f(h), h \leq n$, so $h$ is cancelled. Therefore, $b_{f}(n)>n$.

5. $f\left(n_{1}, n_{2}, \ldots, n_{s}\right)=n_{1} n_{2} \cdot \ldots \cdot n_{s}, s \geq 2$

Let $\left\{p_{t}\right\}_{t=1}^{\infty}$ be an increasing sequence of the prime numbers.

Our aim in this chapter is to find an algorithm which gives only prime numbers.

Theorem 5.1. Let $f: \mathbb{N}^{s} \rightarrow \mathbb{N}, s \geq 2, f\left(n_{1}, n_{2}, \ldots, n_{s}\right)=n_{1} n_{2} \cdot \ldots \cdot n_{s}$. We have:

$$
b_{f}(1)=1, b_{f}(2)=1, \ldots, b_{f}(s-1)=1, b_{f}(s)=2
$$

and if $n>s$, then $b_{f}(n)=p_{t}$, where $t>1$ is chosen in such a way that $p_{t-1} \leq n-s+1<p_{t}$.

Proof. By a straightforward verification, we get (19).

Let $n>s$. We assume that $p_{t-1} \leq n-s+1<p_{t}, t>1$.

We have to prove that $p_{t}$ is non-cancelled, but any natural number $h<p_{t}$ is cancelled.

First, let $p_{t} \mid n_{1} n_{2} \cdot \ldots \cdot n_{s}$ for some $n_{1}, n_{2}, \ldots, n_{s} \in \mathbb{N}$. Thus, there exists a natural $j \leq s$, such that $p_{t} \mid n_{j}$. Since $n_{1}, n_{2}, \ldots, n_{s} \geq 1$ and $n_{j} \geq p_{t}, n_{1}+n_{2}+\cdots+n_{s} \geq p_{t}+s-1>n$. Therefore, a number $p_{t}$ is non-cancelled. 
We now assume that $h<p_{t}$. To show that $h$ is cancelled, we need to consider two cases separately.

a) If $h=p_{j}$, where $j \in \mathbb{N}$ and $j \leq t-1$, then we take $n_{1}=p_{j}, n_{2}=1, \ldots, n_{s}=1$ and get $h \mid n_{1} n_{2} \cdot \ldots \cdot n_{s}$ with $n_{1}+n_{2}+\cdots+n_{s}=s-1+p_{j} \leq s-1+p_{t-1} \leq n$. Thus, $h$ is cancelled.

b) If $h=k l$ where $k, l>1, k, l \in \mathbb{N}$, we have $(k-2)(l-2) \geq 0$. Thus, $k+l \leq \frac{1}{2} k l+2$.

We take $n_{1}=k, n_{2}=l, n_{3}=1, \ldots, n_{s}=1$ and get $h \mid n_{1} n_{2} \cdot \ldots \cdot n_{s}$.

From Bertrand's postulate, we have $p_{t}<2 p_{t-1}$ for $t>1$. Therefore,

$$
\begin{aligned}
& n_{1}+n_{2}+\cdots+n_{s}=k+l+s-2 \leq \frac{1}{2} k l+s=\frac{1}{2} h+s \leq \\
& \frac{1}{2}\left(p_{t}-1\right)+s=\frac{1}{2}\left(p_{t}+1\right)+s-1 \leq p_{t-1}+s-1 \leq n .
\end{aligned}
$$

Thus, $h$ is cancelled.

To summarise, we have shown that every $h<p_{s}$ is cancelled and this is the end of the proof.

Remark 5.2. The set $\left\{b_{f}(n): n>s-1, n \in \mathbb{N}\right\}$ is the set of all prime numbers.

\section{6. $f\left(n_{1}, n_{2}\right)=\operatorname{od}\left(n_{1}+n_{2}\right)-o d\left(n_{1}\right)$, where $\operatorname{od}(n)$ denotes $n$-th odious number}

The 'odious' numbers are the numbers $(o d(n))_{n \geq 1}$ from the sequence A000069 in [14],

$$
1,2,4,7,8,11,13,14,16,19,21,22,25,26,28 \ldots
$$

and this sequence contains consecutive natural numbers with an odd number of digit ' 1 ' in their binary representation.

Remark 6.1. Note that $f(1, n)=f_{n}=o d(n+1)-o d(n)$ gives the Thue-Morse ternary sequence: $1,2,3,1,3,2,1,2,3,2,1,3,1,2,3,1, \ldots$

The idea behind the construction of this sequence is based on the following recursive definition $([14])$ :

1. Start with $f_{1}=1, f_{2}=2$.

2. For any $k \in \mathbb{N}$, we will give a procedure describing how to construct the values $f_{2^{k}+1}, \ldots, f_{2^{k+1}}$ tarting from the values $f_{1}, \ldots, f_{2^{k}}$ :
a) Write: $f_{1}, f_{2}, \ldots, f_{2^{k}}, 4-f_{1}, 4-f_{2}, \ldots, 4-f_{2^{k}}$.
b) Transform the value of $4-f_{2^{k}}$ according to the rule: replace 3 with 2 , replace 2 with 1 , leave 1 unchanged.
c) After transformation, we get the sequence $f_{1}, \ldots, f_{2^{k}}, f_{2^{k}+1}, \ldots, f_{2^{k+1}}$. 
Theorem 6.2. Let $f: \mathbb{N}^{2} \rightarrow \mathbb{N}$, we take the function $f\left(n_{1}, n_{2}\right)=o d\left(n_{1}+n_{2}\right)-o d\left(n_{1}\right)$. Then

Proof. We have:

$$
b_{f}(n)=\min \left\{2^{e}: 2^{e} \geq n\right\} .
$$

$$
\begin{aligned}
& V_{f}(n)=\left\{\operatorname{od}\left(n_{1}+n_{2}\right)-o d\left(n_{1}\right): n_{1}+n_{2} \leq n\right\}= \\
& =\{\operatorname{od}(r)-o d(s): 1 \leq s<r \leq n\} .
\end{aligned}
$$

By definition, $b_{f}(n)$ is the least natural number $m$ in the set $\mathbb{N} \backslash\left\{d \in \mathbb{N}: d \mid a\right.$ for some $\left.a \in V_{f}(n)\right\}$. Thus, $b_{f}(n)$ is the least natural number $m$ that discriminates the numbers $o d(1), o d(2), \ldots, o d(n)$. Taking into account the differences in the definitions of discriminator $D_{g}(n)$ and $b_{f}(n)$, we obtain (20) from Theorem 5 [4].

7. $f\left(n_{1}, n_{2}\right)=\frac{1}{4}\left|3^{n_{1}+n_{2}}-3^{n_{1}}-5\left[(-1)^{n_{1}+n_{2}}-(-1)^{n_{1}}\right]\right|$.

Remark 7.1. The sequence $u_{5}(r):=\frac{1}{4}\left(3^{r}-5(-1)^{r}\right)$ was first investigated by Sabin Sălăjan [15]. Note that $f\left(n_{1}, n_{2}\right)=\left|u_{5}\left(n_{1}+n_{2}\right)-u_{5}\left(n_{1}\right)\right|$.

Theorem 7.2. Let $f: \mathbb{N}^{2} \rightarrow \mathbb{N}$, we take the function:

$$
f\left(n_{1}, n_{2}\right)=\frac{1}{4}\left|3^{n_{1}+n_{2}}-3^{n_{1}}-5\left[(-1)^{n_{1}+n_{2}}-(-1)^{n_{1}}\right]\right| .
$$

Then

$$
b_{f}(n)=\min \left\{2^{e}, 5^{f}: 2^{e} \geq n, 5^{f} \geq \frac{5}{4} n\right\} .
$$

Proof. Note that this theorem is an obvious corollary from Browkin's Conjecture, confirmed by Ciolan and Moree Theorem 2 in [15]. We have:

$$
\begin{aligned}
& V_{f}(n)=\left\{\frac{1}{4}\left|3^{n_{1}+n_{2}}-3^{n_{1}}-5\left[(-1)^{n_{1}+n_{2}}-(-1)^{n_{1}}\right]\right|: n_{1}+n_{2} \leq n\right\}= \\
& \left\{\left|u_{5}(r)-u_{5}(s)\right|: 1 \leq s<r \leq n\right\} .
\end{aligned}
$$

By definition, $b_{f}(n)$ is the least natural number $m$ in the set $\mathbb{N} \backslash\left\{d \in \mathbb{N}: d \mid a\right.$ for some $\left.a \in V_{f}(n)\right\}$. Therefore, $b_{f}(n)$ is the least natural number that discriminates the numbers $u_{5}(1), u_{5}(2), \ldots, u_{5}(n)$. We obtain (22) from Theorem 1 [15]. 
8. $f\left(n_{1}, n_{2}\right)=2^{n_{1}+n_{2}}-2^{n_{1}}$.

In most cases, one can find a discriminator for some special types of functions. There is still lack of an algorithm for any larger class of mappings, for example, all polynomials or all exponential functions. However, now we will describe a simple, general idea of how to find at least an estimation for the discriminator. More accurately, if for any $n \in \mathbb{N}$ we find the set $Z_{n}$ such that $b_{f}(n) \notin Z_{n}$, then we have the lower bound of $b_{f}(n)$ in the form:

$$
b_{f}(n) \geq \min \left\{m: m \notin Z_{n}, m \in \mathbb{N}\right\} .
$$

Obviously, $Z_{n}=\mathbb{N} \backslash\left\{b_{f}(n)\right\}$ satisfies the condition $b_{f}(n) \notin Z_{n}$, but the aim is use properties of $f$ to find $Z_{n} \subset \mathbb{N} \backslash\left\{b_{f}(n)\right\}$ such that

$$
\min \left\{m: m \notin Z_{n}, m \in \mathbb{N}\right\}=\min \left\{m: m \notin \mathbb{N} \backslash\left\{b_{f}(n)\right\}, m \in \mathbb{N}\right\}=b_{f}(n) .
$$

Below, we will follow this line of reasoning for the function

$$
f\left(n_{1}, n_{2}\right)=2^{n_{1}+n_{2}}-2^{n_{1}}
$$

We recall that $\varphi(n)=|\{m \in \mathbb{N}: m \leq n,(m, n)=1\}|$.

Theorem 8.1. Let $f: \mathbb{N}^{2} \rightarrow \mathbb{N}$, we take the function:

Then:

$$
f\left(n_{1}, n_{2}\right)=2^{n_{1}+n_{2}}-2^{n_{1}}
$$

$$
b_{f}(n) \geq \min \{m: \varphi(m) \geq n, m \in \mathbb{N}\}, \text { for } n \geq 2 .
$$

Proof. We assume that $n \geq 2$. Let $Z_{n}:=\{m: \varphi(m) \leq n-1, m \in \mathbb{N}\}$.

Since $\{1,2, \ldots, n\} \subset Z_{n}$, then $Z_{n} \neq \emptyset$. We will show that if $m \in Z_{n}$, then $m$ is cancelled. Let $m=2^{s} m_{1}$, where $m_{1}$ is an odd number, $s \geq 0$.

We take $n_{1}=\max \{s, 1\}$ and $n_{2}=\varphi\left(m_{1}\right)$, then

$$
n_{1}+n_{2}=\max \{s, 1\}+\varphi\left(m_{1}\right) \leq \varphi\left(2^{s}\right)+\varphi\left(m_{1}\right) \leq \varphi\left(2^{s}\right) \varphi\left(m_{1}\right)+1=\varphi(m)+1 \leq n .
$$

Thus, $2^{n_{1}+n_{2}}-2^{n_{1}} \in V_{f}(n)$. Note that $m \in \Delta_{f}(n)$. Indeed, by Euler's theorem:

$$
2^{n_{1}+n_{2}}-2^{n_{1}}=2^{\varphi\left(m_{1}\right)+\max \{s, 1\}}-2^{\max \{s, 1\}}=2^{\max \{s, 1\}}\left(2^{\varphi\left(m_{1}\right)}-1\right) \equiv 0(\bmod m) \text {, so } m \notin \mathbb{N} \backslash \Delta_{f}(n) .
$$

Thus, $m$ is cancelled. Therefore, $b_{f}(n) \notin Z_{n}$ and $\varphi\left(b_{f}(n)\right) \geq n$, which implies (25).

Comment. Now we are going to prove the theorem which allows us to rewrite $(25)$ in a simpler form. However, the proof depends on some conjecture still waiting to be proved. 
Remark. We will recall Sierpiński's conjecture, which states that for every integer $x>1$ there is at least one prime number in $\left(x^{2}-x, x^{2}\right)$, see [9]. However, many similarsounding conjectures have appeared over the years. Oppermann [19] stated the conjecture that for every natural $x>1$, the interval $\left(x^{2}-2 x+1, x^{2}\right)$ contains a prime. Cramer [21] stated the conjecture that for every $n$-th prime number $p_{n}$, we have $p_{n+1}-p_{n}=O\left(\left(\log p_{n}\right)^{2}\right)$. Baker, Harman and Pintz proved that for sufficiently large real number $x$, there is at least one prime number in $\left[x-x^{0.525}, x\right]$, see $[18]$. For a brief review of other theorems and conjectures about $p_{n+1}-p_{n}$, we refer the reader to paper $[16]$.

\section{Conjecture 8.2.}

$$
\min \{m: \varphi(m) \geq n, m \in \mathbb{N}\}=\min \{p: p>n, p \text { is a prime number }\} .
$$

Theorem. If Sierpiński's conjecture is true, then (27) is also true.

Proof. First, we will prove an inequality without using any conjecture.

$$
\min \{m: \varphi(m) \geq n, m \in \mathbb{N}\} \leq \min \{p: p>n, p \text { is a prime number }\} .
$$

If $p$ is a prime number such that $p>n$, then $\varphi(p)=p-1 \geq n$.

Let $k=\min \{p: p>n, p$ is a prime number $\}$.

Hence, $\varphi(k) \geq n$, so $k \in\{m: \varphi(m) \geq n, m \in \mathbb{N}\}$ and

$$
\min \{p: p>n, p \text { is a prime number }\} \geq \min \{m: \varphi(m) \geq n, m \in \mathbb{N}\} .
$$

Secondly, we will prove the opposite inequality using Sierpiński's conjecture.

$$
\min \{m: \varphi(m) \geq n, m \in \mathbb{N}\} \geq \min \{p: p>n, p \text { is a prime number }\} .
$$

We start this proof using the fact that for every composite number $m$, we have (see [8]):

$$
\varphi(m) \leq m-\sqrt{m} .
$$

Suppose that $m_{0}=\min \{m: \varphi(m) \geq n, m \in \mathbb{N}\}$ is a composite number.

If Sierpiński's conjecture is true, then there exists prime number $p$ such that

$$
n \leq \varphi\left(m_{0}\right) \leq m_{0}-\sqrt{m_{0}}<p<m_{0} .
$$

Therefore, by (28) we get $p \geq \min \{m: \varphi(m) \geq n, m \in \mathbb{N}\}=m_{0}$, which leads to the contrary.

It means that $m_{0}$ is a prime, so $m_{0} \geq \min \{p: p>n, p$ is a prime number $\}$.

\section{The Fibonacci numbers $F_{a}$}

Theorem 9.1. For the Fibonacci numbers $F_{a}$, we take a function $F: \mathbb{N} \rightarrow \mathbb{N}$ such that $F(a)=F_{a}$. Then $b_{F}(6 n) \geq n+1$, if $n$ is a natural number.

Proof. We have $V_{F}(n)=\left\{F_{a}: a \leq n\right\}$ and $\Delta_{F}(n)=\left\{d \in \mathbb{N}: d \mid a\right.$ for some $\left.a \in V_{F}(n)\right\}$. 
It has been proven (see [17]) that for any natural number $m$ the sequence $\left(F_{i} \bmod m\right)_{i=0}^{\infty}$ is periodic with its length being not greater than $6 m$. Thus, for every natural number $k \geq 1$ there exists a natural number $i \leq 6 k$ such that $k \mid F_{i}$. Therefore:

$$
k \in \Delta_{F}(6 k) \text {, }
$$

Thus:

$$
\{1,2, \ldots, n\} \subseteq \Delta_{F}(6 n)
$$

However, by the definition $b_{F}(6 n) \notin \Delta_{F}(6 n)$. Thus, $b_{F}(6 n) \geq n+1$.

\section{0. $b_{f}(n)=f(n)+1$ for surjective, non-decreasing natural-valued arithmetic $f$}

Theorem 10.1. Let $f: \mathbb{N} \rightarrow \mathbb{N}$, such that $f$ is a surjective, non-decreasing function. Then, for all $n \in \mathbb{N}$ we have $b_{f}(n)=f(n)+1$.

Proof. We will prove that $V_{f}(n)=\Delta_{f}(n)=\{f(1), \ldots, f(n)\}, b_{f}(n)=f(n)+1$.

We proceed by induction on $n$. Observe that when $n=1$, we have

$$
f(1)=1, V_{f}(1)=\Delta_{f}(1)=\{1\}, b_{f}(1)=2 .
$$

Assume that the proposition holds for $n-1$, where $n \geq 2$, i.e.

$$
V_{f}(n-1)=\Delta_{f}(n-1)=\{f(1), \ldots, f(n-1)\}, b_{f}(n-1)=f(n-1)+1 .
$$

If $f(n)=f(n-1)$, then $V_{f}(n)=V_{f}(n-1)=\Delta_{f}(n-1)=\Delta_{f}(n), b_{f}(n)=b_{f}(n-1)=$

$$
f(n-1)+1=f(n)+1
$$

If $f(n)=f(n-1)+1$, then $V_{f}(n)=V_{f}(n-1) \cup\{f(n)\}=\{f(1), \ldots, f(n-1), f(n)\}$,

$$
\Delta_{f}(n)=\Delta_{f}(n-1) \cup\{d \in \mathbb{N}: d \mid f(n)\}=\{f(1), \ldots, f(n-1), f(n)\} .
$$

and $b_{f}(n)=f(n)+1$.

Thus, the proposition holds for $n$ and this completes the proof.

We would like to thank the referee for providing useful comments which serve to improve the paper, especially Theorem 9.1.

\section{References}

[1] Arnold L.K., Benkoski S.J., McCabe B.J., The discriminator (a simple application of Bertrand's postulate), Amer. Math. Monthly, 92, 1985, 275-277.

[2] Bremser P. S., Schumer P.D., Washington L.C., A note on the incon-gruence of consecutive integers to a fixed power, J. Number Theory 35, no. 1, 1990, 105-108.

[3] Browkin J., Cao H-Q. Modifications of the Eratosthenes sieve, Colloq. Math., 135, 2014, 127-138. 
[4] Haque S., Shallit J., Discriminators and k-regular sequences, INTEGERS, 16, 2016, Paper A76.

[5] Moree P., Mullen G.L., Dickson polynomial discriminators, J. Number Theory, 59, 1996, 88-105.

[6] Moree P., The incongruence of consecutive values of polynomials, Finite Fields Appl., 2, 1996, 321-335.

[7] Moree P., Zumalacarregui A., Sălăjan's conjecture on discriminating terms in an exponential sequence, J. Number Theory, 160, 2016, 646-665.

[8] Sierpiński W., Elementary theory of numbers, Warszawa 1964.

[9] Sierpiński W., Sur certaines hypothèses concernant les nombres premiers, Acta Arith., 4, no. 1, 1958, 20-30.

[10] Zhi-Wei Sun, On functions taking only prime values, J. Number Theory, 133, 2013, 2794-2812.

[11] Tomski A., Zakarczemny M., On some cancellation algorithms, NNTDM, 23, 2017, 101-114.

[12] Zakarczemny M., On some cancellation algorithms, II, Technical Transactions, vol. 5/2017.

[13] Zieve M., A note on the discriminator, J. Number Theory, 73, 1998, 122-138.

[14] Sloane N.J., The On-Line Encyclopedia of Integer Sequences, available online at https://oeis.org (access: 05.10.2018).

[15] Ciolan A., Moree P., Browkin's Discriminator Conjecture, 2017, available online at https://arxiv.org/abs/1707.02183 (access: 05.10.2018).

[16] Melfi G., On the conditional infiniteness of primitive weird numbers, J. Number Theory, $147,2015,508-514$.

[17] https://webspace.ship.edu/msrenault/fibonacci/fib.htm (access: 05.10.2018).

[18] https://www.mathpages.com/ home/kmath078/kmath078.htm (access: 05.10.2018).

[19] Baker R.C., Harman G., Pintz J., The difference between consecutive primes, II, Proc. Lond. Math Soc., 83, 2001, 532-562.

[20] Oppermann L., Om vor Kundskab om Primtallenes Moengde mellem givne Groenser, Overs. Dansk. Vidensk. Selsk. Forh., 1882, 169-179.

[21] Cramer H., On the order of the magnitude of the difference between consecutive prime number, Acta Arith. 2, 1936, 23-46. 